\title{
NMO-IgG: A specific biomarker for neuromyelitis optica
}

\author{
Brian G. Weinshenker ${ }^{\mathrm{a}, *}$, Dean M. Wingerchuk ${ }^{\mathrm{d}}$, Sean J. Pittock ${ }^{\mathrm{a}, \mathrm{b}}$, Claudia F. Lucchinetti ${ }^{\mathrm{a}}$ and \\ Vanda A. Lennon ${ }^{\mathrm{a}, \mathrm{b}, \mathrm{c}}$ \\ ${ }^{a}$ Department of Neurology, Mayo Clinic College of Medicine, Rochester MN, USA \\ ${ }^{\mathrm{b}}$ Laboratory Medicine and Pathology, Mayo Clinic College of Medicine, Rochester MN, USA \\ ${ }^{\mathrm{c}}$ Department of Immunology, Mayo Clinic College of Medicine, Rochester MN, USA \\ ${ }^{\mathrm{d}}$ Department of Neurology, Mayo Clinic Scottsdale, AZ, USA
}

\begin{abstract}
Neuromyelitis optica (NMO) is an inflammatory demyelinating disease that principally targets the optic nerves and spinal cord and often leads to severe disability and occasionally life threatening respiratory failure. Although its clinical manifestations overlap with those of multiple sclerosis (MS), in established cases these two conditions can be distinguished on the basis of clinical, radiological, and routine spinal fluid studies. The diagnosis in early cases or limited forms of NMO is difficult. We recently discovered a unique IgG autoantibody (NMO-IgG) that is highly specific to patients with NMO and thus a valuable diagnostic aid. Its antigen, aquaporin-4 (AQP4), is the central nervous system's predominant water channel protein. This antibody has not yet been proven to be pathogenic, but several facts suggest that it might be, including the similarity of the immunohistochemical pattern of NMO-(AQP4) IgG binding to mouse CNS tissues to the pattern of immune complex deposition in autopsied patients' spinal cord tissue. The spectrum of diseases identified by NMO-IgG is broader than has previously been recognized clinically and includes incomplete forms of NMO, such as recurrent transverse myelitis without optic neuritis and recurrent optic neuritis without myelitis.
\end{abstract}

Keywords: Neuromyelitis optica, transverse myelitis, optic neuritis, biomarker, autoantibody, immunofluorescence

\section{Introduction}

Neuromyelitis optica (NMO) is an inflammatory demyelinating disease of the central nervous system (CNS), with clinical manifestations resembling multiple sclerosis (MS) [1-3]. The principal characteristics that distinguish NMO from MS are its selective involvement of optic nerves and spinal cord, and its characteristic longitudinally extensive spinal cord lesions that are detected most sensitively by magnetic resonance imaging (MRI). NMO is considered synonymous with Devic's disease. Before the advent of a serological marker specific for NMO, these observations validated Devic's

${ }^{*}$ Corresponding author: Dr. Brian Weinshenker, Mayo Clinic College of Medicine, 200 First St. SW, Rochester MN 55905, USA. Tel.: +1 507538 1039; Fax: +1 5072664419 ; E-mail: weinb@mayo.edu. perceptive conclusion that NMO was distinct from prototypic MS. For many years NMO had been diagnosed in western countries in accordance with Devic's traditional criteria, namely in individuals with bilateral optic neuritis and myelitis that develop in quick succession. However, individual neurologists have defined the allowable interval between index episodes differently and arbitrarily. In the past 7 years, the clinical definition of NMO has been expanded beyond Devic's clinical descriptions, which were based on relatively few cases. More liberal diagnostic criteria include patients with unilateral optic neuritis and do not limit the interval between the index episodes [1]. However, to ensure specificity of the clinical diagnosis, the expanded diagnostic criteria were more restrictive by requiring MRI demonstration of a longitudinally extensive cord lesion or lack of brain lesions. Patients with NMO defined by either the more traditional or more liberal criteria, 
have a similar course, outcome and response to treatment, and differ in their course from patients assigned a diagnosis of MS.

In this review, we outline the key clinical and pathological characteristics that define NMO, and describe the discovery of a novel marker autoantibody ("NMOIgG" defined immunohistochemically by the staining pattern yielded by a patient's serum IgG binding to a section of mouse brain tissue) and its unique class of autoantigen. The autoantibody staining pattern is highly specific for NMO and, when positive $(70 \%$ of cases), distinguishes NMO from MS [4]. Detection of this autoantibody facilitates the diagnosis of NMO at an early point in the disease, before fulfillment of all traditional clinical diagnostic criteria, and when clinical distinction from MS is difficult and sometimes impossible. Seropositivity for NMO-IgG supports the diagnosis in atypical cases and in patients who have recurrent myelitis alone or recurrent optic neuritis alone as a limited manifestation of NMO. These patients are excluded from the diagnostic category of NMO by criteria proposed in 1999 (Table 1). Our group was challenged to validate a biomarker for NMO in the absence of a "gold standard" for the clinical diagnosis. We had to contend with the issue of "circular logic". However, by insisting on the presence of the cardinal features of NMO, especially those that are most distinct from MS, such as the occurrence of a longitudinally extensive lesion in the spinal cord, and by conducting blinded evaluations of the serological marker using the key specific elements of the 1999 clinical diagnostic criteria, we have established the disease specificity of NMO-IgG convincingly.

\section{Comparative clinical features NMO and MS}

The coexistence of optic nerve and spinal cord dysfunction was first described by Albutt in the late 19th century. In 1894, Gault used the term neuromyélite optic aiguё (acute optic neuromyelitis) to describe 17 cases collected from the literature and personal experience by his mentor, Eugene Devic. From then on, the disorder was known also as Devic's disease or Devic's syndrome $[5,6]$. The case descriptions had substantial impact because the resulting traditional definition of NMO - an acute, fulminant, monophasic disorder consisting of optic neuritis and myelitis occurring simultaneously or in rapid succession - still lingers in modern neurology texts, along with debate regarding the relationship of NMO to MS.
Despite its apparent clarity, the nosology and definition of NMO has been debated frequently. Cases with "atypical" features, such as a relapsing course, were described in the 1930s [7] and fueled debate about whether NMO was anything other than an unusually severe MS variant. Contemporary authors have expanded the NMO spectrum by developing arbitrary definitions, based on case series, that seemed to describe a group of patients quite different than those with MS [1,3]. The expanded definition allows any of the following features: 1) unilateral optic neuritis; 2) elimination of the arbitrary interval over which the first events of optic neuritis and myelitis occur; and 3) a relapsing course. Technological innovations such as immunochemical analysis of cerebrospinal fluid (CSF) and magnetic resonance imaging (MRI) (see below) have allowed further criterion refinements but many patients whom clinicians suspect have "possible" NMO cannot be diagnosed using existing criteria. These diagnostic considerations, reinforced by the recognition that NMO was very similar to the Asian optic-spinal form of MS [8,9], and the clinical experience that optimal therapies for NMO and MS differ [10], provided impetus for seeking a biomarker to distinguish these two disorders.

\subsection{Epidemiology}

NMO typically affects middle-aged women, with predilection for non-Caucasians, but infantile and geriatric cases have been reported. The female-to-male ratio approximates 7:1 for the relapsing form of the disease and the median age of onset is 40, roughly one decade older than for typical MS [3]. A relatively uncommon monophasic form of NMO, occurring in men and women at the same frequency, is characterized by almost simultaneous optic neuritis, often bilateral, and myelitis. The incidence and prevalence of NMO are unknown. In Western nations, it has been considered a rare disorder but, in our evolving experience, it is clearly under-recognized and usually misdiagnosed as MS. Approximately 100 cases have been seen in the Department of Neurology, Mayo Clinic in Rochester MN over the past 5 years. The proportion of CNS demyelinating disease accounted for by NMO is much higher in non-Caucasians (e.g., African, Hispanic, North American Indian, and Asian ethnic groups) than it is for MS. In Japan, the optic-spinal form of MS may account for $30 \%$ of CNS demyelinating disease cases [8].

The genetic factors predisposing to NMO are unknown. Most cases appear to be sporadic but identical twins [11] and affected female siblings [12,13] 
Table 1

Diagnostic criteria for neuromyelitis optica [1]

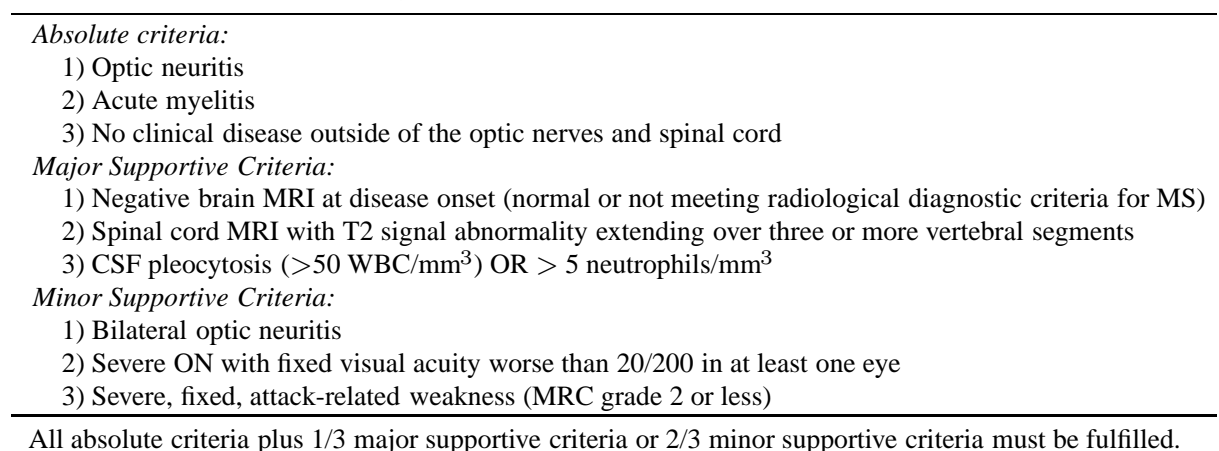

have been reported. The class II major histocompatibility complex allele $D P B 1 * 0501$ is associated with optic-spinal MS in Japanese patients, whereas "Western" MS in Japanese patients is most consistently associated with $D R B 1 * 1501$ [14].

\subsection{Clinical characteristics and diagnosis}

Diagnostic criteria for NMO are summarized in Table 1. Patients present with unilateral or bilateral optic neuritis, acute myelitis, or a combination of the two events. Spinal cord attacks usually present as "complete" transverse myelitis with bilateral motor weakness, a bilaterally symmetric sensory level, sphincter dysfunction, pain, paroxysmal tonic spasms, and incomplete recovery. In contrast, most MS-related myelitis attacks are milder "incomplete" or "partial" cord syndromes associated with unilateral or asymmetric weakness and sensory symptoms. Nonetheless, myelitis in NMO may be clinically complete, almost complete or incomplete. Optic neuritis attacks are similar to those associated with MS but tend to be more severe and leave a greater residual deficit. Neurological symptoms referable to CNS sites other than the optic nerves and spinal cord are uncommon and before 2006 we excluded patients with syndromes involving other regions of the CNS to achieve a more clear distinction between NMO and MS in the criteria we proposed.

The diagnosis of NMO may be supported by integrating laboratory and neuroimaging data. Spinal fluid analyses reveal oligoclonal IgG bands in about $35 \%$ of NMO patients, compared with 80-90\% of patients with MS [3]. Unlike the situation in MS, the CSF leukocyte count in NMO may exceed 50 million leukocytes/L, and polymorphonuclear cells sometimes predominate in the first weeks following the onset of an acute myelitis event. More than half of NMO patients have either a coexisting autoimmune disorder (most commonly thy- roid disorders, but also a broad range of organ-specific and non-organ-specific autoimmune disorders) or multiple serum autoantibodies such as antinuclear antibodies, especially those of extractable nuclear antigen specificity [3,15]. Cranial MRI is generally normal or reveals some nonspecific white matter abnormalities that do not fulfill radiological criteria for MS [3]. Serial cranial MRI studies over several years may demonstrate an increasing burden of white matter lesions but in only $10 \%$ of cases are criteria for MS satisfied. An additional $8 \%$ of cases may develop "MS-atypical lesions", including brainstem and hypothalamic lesions, or large fluffy hemispheric lesions; certain brainstem and hypothalamic lesions are now recognized as being specific for NMO [16]. Optic nerve enhancement can support a clinical diagnosis of optic neuritis but is not specific.

Spinal cord MRI has emerged as the most discriminating laboratory test. Virtually all patients with a clinical diagnosis of NMO have a longitudinally extensive cord lesion extending over three or more vertebral segments; such lesions are usually located in the central cord and enhance after gadolinium administration. In contrast, spinal MS plaques are usually less than one vertebral segment in craniocaudal length and are asymmetric.

\subsection{Natural history and course}

Contrary to the historical definition of NMO, more than $80 \%$ of affected patients follow a relapsing course with recurrent attacks of optic neuritis, myelitis, or both. Although initial attacks are more severe in patients who prove to have monophasic NMO, long-term neurological prognosis is somewhat better due to absence of new attacks. Five-year survival is approximately $90 \%$ for patients with monophasic disease and 
less than $80 \%$ for patients with relapsing disease [3, 17].

Relapsing NMO has an unpredictable course with clusters of attacks occurring months or years apart. Relapses occur early: $55 \%$ within one year, $78 \%$ by three years and $90 \%$ by five years. More than $50 \%$ of patients have monocular blindness or cannot ambulate without assistance five years after disease onset, a much worse outcome than for classical MS. Most of the more than $20 \%$ of patients who die in the first 5 years succumb to respiratory failure complicating a severe attack of cervical myelitis. Some clinical features, such as a greater interval between the first and second index attacks, female sex, and better motor recovery after the first myelitis attack, are independently associated with a subsequent relapsing rather than monophasic course. MRI and CSF characteristics do not predict disease course.

The diagnostic and therapeutic challenges presented by patients with an initial episode of optic neuritis or myelitis emphasize the importance of having a specific biomarker to enable early diagnosis of NMO. Physicians familiar with the clinical, laboratory and imaging characteristics by which it may be distinguished from MS may diagnose an established case of NMO with relative ease. However, NMO typically presents with optic neuritis or longitudinally extensive transverse myelitis. There is a broad differential diagnosis for each of these syndromes, and it is currently impossible to reliably distinguish optic neuritis due to MS from that due to NMO, or to distinguish transverse myelitis occurring as a first event of NMO from "idiopathic" transverse myelitis. Also, it would be desirable to differentiate relapsing from monophasic NMO at disease onset because only those with relapsing disease require preventative immunotherapy. A biomarker for the relapsing form would facilitate early diagnosis, possibly at the time of the first attack, and optimally would be able to predict relapsing disease. This would substantially impact the choice of treatment which differs for NMO (immunosuppression) and MS (immunomodulation with beta-interferon or glatiramer acetate).

\section{The pathology and immunology of NMO}

Lesions are generally restricted to the spinal cord and optic nerves, and involve white matter and gray matter. Acute spinal cord lesions demonstrate patchy or continuous swelling and softening extending over multiple spinal segments. Active lesions in both op- tic nerve and spinal cord are characterized by infiltrating macrophages and activated microglia, plus demyelination, axonal loss, and prominent necrosis [18]. (Fig. 1A) Perivascular inflammation is variable. Chronic lesions are characterized by gliosis, cystic degeneration, cavitation, and atrophy. Blood vessels within demyelinating lesions are distinctively thickened, and hyalinized [18]. While NMO lesions share many characteristics of MS lesions (sharply circumscribed, $\mathrm{T}$ cell and macrophage infiltration), the distinctive features in NMO include the restricted topographic distribution of the lesions, severe necrosis, hyalinization of blood vessels, prominent infiltration by eosinophils and neutrophils, and peculiar pattern of antibody deposition and complement activation.

The selective involvement of optic nerves and spinal cord in NMO is not understood. A detailed neuropathological analysis of more than 80 spinal cord lesions from a total of nine autopsy cases suggests targeting of the perivascular region by an antibodydependent, complement-mediated attack, with further amplification by the recruitment and degranulation of eosinophils [19]. Perivascular deposits of immunoglobulin and products of complement activation are found in all actively demyelinating NMO lesions (Fig. 1B). Complement components and immunoglobulins are distributed in characteristic ring and rosette patterns surrounding penetrating microvessels (Fig. 1C-F). Complement activation, eosinophilic infiltration, and vascular fibrosis are more prominent in NMO lesions than in MS lesions. Macrophage infiltration and microglial activation are evident in acute MS lesions, but gray matter involvement, infiltration of eosinophils or other granulocytes, and the severe necrosis typical of NMO lesions are rarely seen in MS lesions. Furthermore, although deposits of complement and immunoglobulin are a characteristic of the subset of MS lesions classified by Lucchinetti et al. [20] as "pattern 2", they are at sites of active myelin destruction and not prominently perivascular in their distribution as they are in NMO lesions.

The pathology of NMO resembles that of experimental autoimmune encephalomyelitis (EAE) induced in the Brown Norway (BN) strain of rat by immunization with soluble myelin oligodendrocyte glycoprotein (MOG) in Freund's adjuvant [21,22]. This form of EAE has a predilection for the optic nerves and spinal cord; active demyelination is associated with prominent deposition of antibody and products of complement activation; and the inflammatory infiltrate contains large numbers of eosinophils. 


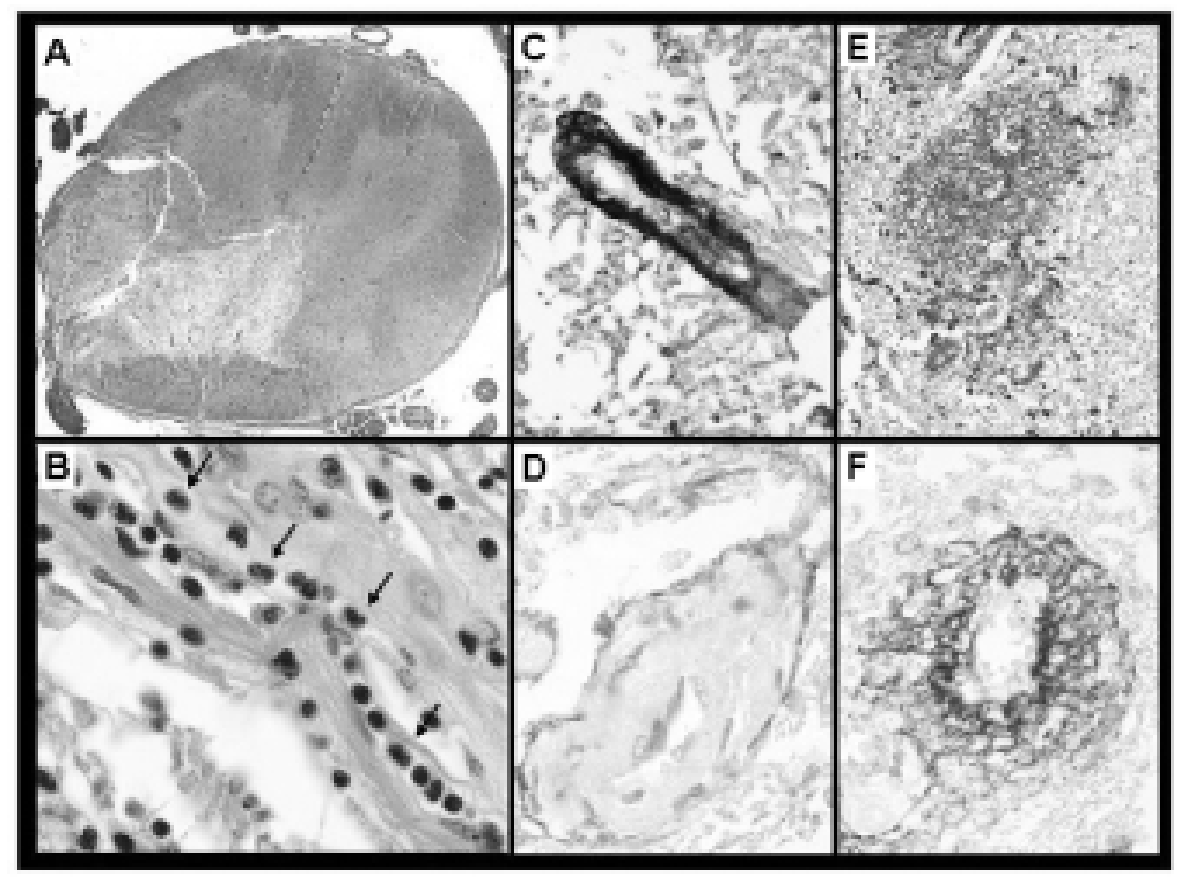

Fig. 1. Pathological Features of NMO. (Modified by permission from C.F. Lucchinetti, R.N. Mandler, D. McGavern, W. Bruck, G. Gleich, R.M. Ransohoff, C. Trebst, B. Weinshenker, D. Wingerchuk, J.E. Parisi, H. Lassmann. A role for humoral mechanisms in the pathogenesis of Devic's neuromyelitis optica. Brain. 2002; 125:1450-1461.) A. Spinal cord cross-section demonstrating extensive demyelination involving both the gray and white matter (Luxol fast blue and PAS myelin stain). B. Numerous perivascular eosinophils are located within the lesion (hematoxylin eosin) C. There is pronounced immunoglobulin reactivity (human Ig); and complement activation in a perivascular rim pattern (D) (C9neo antigen (red)). E: Immunocytochemistry for IgM demonstrates a rosette perivascular staining pattern, and staining for complement activation with C9neo-antigen (red) demonstrates a similar rosette $(\mathrm{F})$ pattern of staining.

The pathology of NMO and its similarities to MOGinduced $\mathrm{EAE}$ in the $\mathrm{BN}$ rat [22] support a central role for humoral effector mechanisms in the pathogenesis of NMO. The vasculocentric distribution of antibody deposition and complement activation in NMO suggests that the primary target of a pathogenic autoantibody is located in the perivascular region.

\section{Discovery of NMO-IgG: Pattern of binding in mouse brain and clinical correlations}

Recognition in the late 1990's that patients with NMO frequently have multiple autoimmune disorders and a diversity of autoantibodies, prompted our search for a specific autoantibody marker of NMO. We were particularly impressed by the coexistence of NMO with organ-specific autoimmune disorders, which historically were the first clue that myasthenia gravis [23] and the Lambert-Eaton syndrome [24] were autoimmune disorders. Thus, in 1998, we began to screen serum of patients in whom a diagnosis of NMO was established or who had a syndrome that suggested high risk for future development of NMO (e.g. recurrent longitudinally extensive transverse myelitis, or recurrent optic neuritis particularly in patients lacking MRI brain lesions typical of MS). Sera were evaluated for evidence that IgG might bind selectively to CNS tissues. The assay that we used was optimized in Mayo Clinic's Neuroimmunology Laboratory in 1994 to detect neural-restricted IgG autoantibodies in patients with paraneoplastic autoimmunity [25].

After testing the first few NMO patients' sera blinded, a recurring pattern was noted to be identical to that of an unclassified IgG of unknown clinical significance that the Neuroimmunology Laboratory had documented photographically in the preceding 2 years [4] (Fig. 2). Unmasking of the initial few blinded NMO and control patients' sera confirmed an association between the distinctive IgG staining pattern and the clinical diagnosis of NMO. We therefore retrospectively requested clinical information for the seropositive patients whose undiagnosed neurological disorder had prompted submission of their serum to Mayo Clinic's Neuroimmunology Laboratory for paraneoplastic autoantibody evaluation. Review of this in- 

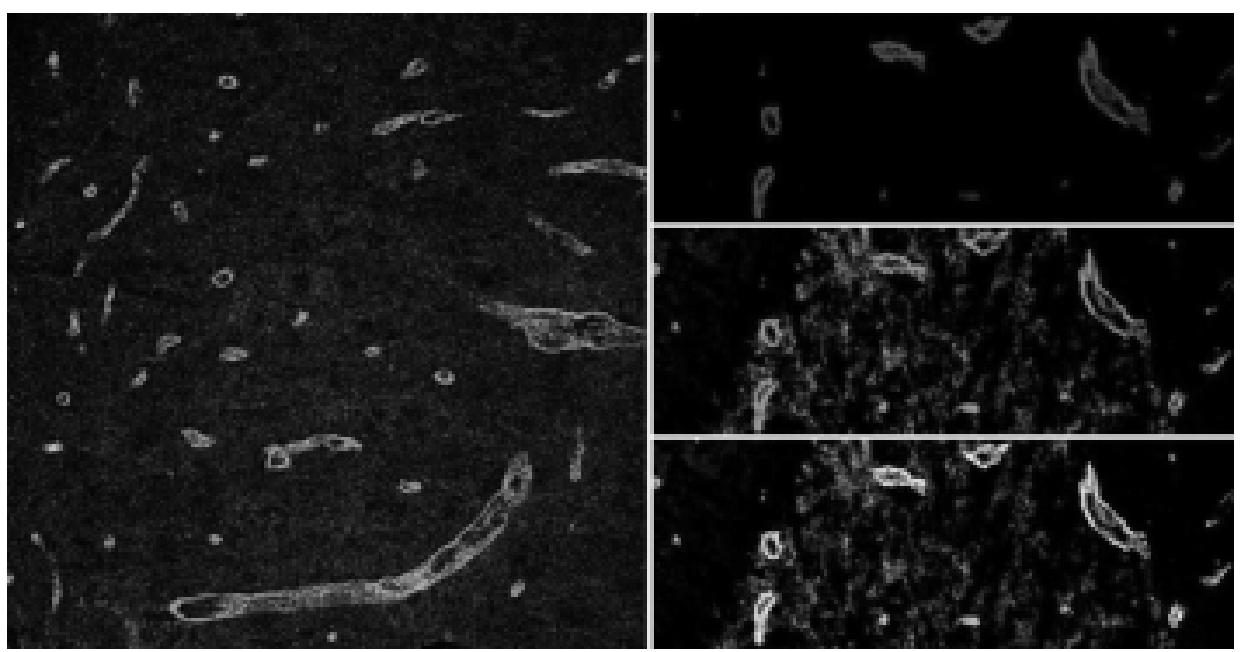

Fig. 2. NMO-IgG pattern: Dual immunofluorescence staining of mouse spinal cord and cerebellar cortex. (Reprinted with permission from Lennon VA et al., A serum autoantibody marker of neuromyelitis optica. Lancet 364: 2106-2112, 2004). NMO-IgG binds in a perivascular location to the AQP4 water channel, which is a component of the dystroglycan protein complex, as is the extracellular matrix protein laminin. Endothelium of spinal cord microvessels (left panel) binds rabbit IgG specific for factor VIII (red). Microvessel profiles in cerebellar granular layer and white matter (right panel) are stained red by rabbit anti-laminin IgG (top right) and green by NMO-IgG (middle right). The yellow stain in merged images (bottom right) indicates close proximity of laminin to the AQP4 target of NMO-IgG in astrocytic foot processes. Fig. 1.

Table 2

Results of Serological Surveys with NMO-IgG

\begin{tabular}{lrrc}
\hline & + & - & Total \\
\hline Definite NMO $^{*}$ & 33 & 12 & 45 \\
High risk syndrome* $^{*}$ & 16 & 19 & 35 \\
MS-NMO mimics $^{\dagger}$ & 2 & 20 & 22 \\
Japanese optic-spinal MS $_{\text {Japanese "Western" MS }}$ & 7 & 5 & 12 \\
Classical MS & 0 & 5 & 5 \\
Miscellaneous $^{\ddagger}$ & 0 & 19 & 19 \\
\hline
\end{tabular}

* Single or recurrent longitudinally extensive myelitis or recurrent optic neuritis with negative or nonspecific findings on MRI of the head.

†Optic neuritis, myelitis (generally not longitudinally extensive), negative MRI of the head; final diagnosis was MS rather than NMO. $\ddagger$ Autoimmune, paraneoplastic and other neurological disease controls.

formation revealed that each patient had a clinical presentation of subacute or relapsing myelopathy with or without vision impairment, compatible with a clinical diagnosis of NMO. We ultimately discovered that 12 of the first 14 individuals ascertained by detection of this distinctive pattern of $\mathrm{IgG}$ binding by personnel in the clinical service laboratory, without any knowledge of the clinical history, had symptoms consistent with NMO (longitudinally extensive myelitis, optic neuritis or both).

We recognized further that the pattern of patients' $\operatorname{IgG}$ binding to the outer face of penetrating microvessels in the mouse brain substrate was similar to the subsequently published immunohistochemical pattern of immunoglobulin and complement deposition detected around small vessels in a Mayo Clinic study of autopsied spinal cord tissues of patients with NMO [19]. Confident that these observations meshed intellectually, we resumed our blinded study with a larger scale prospective survey of sera from patients with clinically definite NMO or a syndrome deemed to be at high risk for progression to NMO, and two independent readers who were blind to diagnoses [4]. Their scores were $100 \%$ concordant. Any serum that yielded the staining pattern characteristic of NMO-IgG at initial screening was scored positive, and retested (again blinded) to confirm its detection, and titrated to the endpoint of detection. No serum was scored "indeterminate" or "equivocal". In the cerebellum and midbrain, NMO$\mathrm{IgG}$ bound in a linear pattern to juxtaposed pial membranes (pia, pial lining of Virchow-Robin spaces) with continuation along microvessels. There was a meshlike staining of white matter and the subpial region. Thirty three (73\%) of 45 patients with clinically defined NMO were seropositive. We included patients with classical MS that initially involved spinal cord and optic nerves, and patients with paraneoplastic autoimmune disorders, assorted myelopathies and miscellaneous disorders as neurological control groups. Two of 22 patients who presented with optic neuritis or myelitis (in most cases not longitudinally extensive) and were judged at the study's end to have MS were seropositive for NMO-IgG. The final diagnosis for those two 
seropositive patients would be classified today as NMO, but the presence of brain lesions on cranial MRI scan mandated a clinical diagnosis of MS for the purpose of our initial report [4]. The test for NMO-IgG had sensitivity of $73 \%$ and specificity of $91 \%$ in distinguishing NMO from MS. None of 19 patients with classical MS and none of 56 individuals with other neurological or systemic autoimmune diseases were positive.

After correlating our observations for North American patients, the data were so persuasive that we extended the survey to study coded sera from patients with Asian optic-spinal form of MS (and local controls), in collaboration with investigators at Tohoku University Medical Center in Sendai, Japan. The goals of this study were to determine whether, as had long been speculated but not proven, this entity is the same as NMO in western populations. The results confirmed the specificity of the findings in the North American population. Specifically, 7 of 12 patients with the opticspinal form of MS in Japan were seropositive versus 0 of 5 patients with classical "Western" MS and 0 of 5 control individuals with cerebral infarcts. The sensitivity $(58 \%)$ and specificity $(100 \%)$ were comparable to our observations in North American patients with NMO [4].

The results of the initial study are summarized in Table 2. We do not have longitudinal data for these patients, and some patients had serum drawn several years after onset of symptoms, so we cannot be sure when NMO-IgG appeared in the serum in relation to disease onset. Some patients were seropositive at the first and thus far only episode of transverse myelitis, which suggests that this marker develops in many patients at an early point in the disease at which time the diagnosis may be uncertain. Anecdotally, we have observed in several patients a progressive increase in NMO-IgG titer prior to relapse, and a progressive reduction in titer in the setting of immunosuppressant therapy, but our longitudinal data are limited and preliminary. At this time, the correlation of NMO-IgG with disease severity or attack severity is unknown.

\section{The spectrum of neurological disorders in which NMO-IgG has been identified}

Once we established the specificity of NMO-IgG in clinically definite cases, the study of patients with clinically similar conditions was appropriate. Our observations thus far suggest that the spectrum of NMO is more broad than previously recognized. In particular, seropositivity for NMO-IgG has allowed us to define "NMO-related diseases" in four situations:
1. limited forms of the disease, e.g. recurrent myelitis without evidence of optic neuritis; recurrent optic neuritis without evidence of myelitis;

2. atypical cases, e.g., patients with clinical or subclinical (MRI) brain involvement, for whom a reliable diagnosis of NMO was not possible based on the 1999 diagnostic criteria;

3. patients with significant comorbidities, particularly connective tissue diseases, in which it had been unclear whether the neurological manifestations were those of NMO or a vasculitic neurological complication of the connective tissue disease;

4. Asian optic-spinal MS, which was recognized as being very similar to NMO; however, clinical differences were cited, the significance of which was uncertain.

Importantly, the seroprevalence of NMO-IgG in patients with recurrent transverse myelitis is thus far as high as in those with definite NMO. In a prospective study of 29 consecutive patients evaluated at Mayo Clinic with a single event of longitudinally extensive "idiopathic" transverse myelitis, 40 percent were seropositive. Of 9 seropositive cases followed for 1 year or longer, $55 \%$ (5 patients) had relapsed (4 patients) or developed optic neuritis (1 patient) within 1 year of follow-up; an additional seropositive patient developed optic neuritis in the second year of followup. In contrast, no seronegative patient had a subsequent neurological event [26]. We have observed clinically silent chiasm lesions at autopsy in patients who have died of recurrent transverse myelitis complicated by respiratory failure, suggesting that on rare occasions involvement of the chiasm/prechiasmatic optic apparatus can occur without symptoms of optic neuritis.

We have conducted a follow-up survey of 72 patients who presented with two or more sequential events of ON without other clinical manifestations that would support a diagnosis of MS or NMO after the second episode. A survival analysis revealed that $12 \%$ developed NMO and 14\% developed MS at 5 years from their first episode of ON. While the risk of converting to MS continued to increase beyond 5 years, the risk of conversion to NMO seemed to end at that point. Approximately half the patients at the final follow-up still carried the diagnosis of "idiopathic recurrent ON" [27]. Of 8 individuals with recurrent $\mathrm{ON}$ tested for NMOIgG prior to 2004, 2 (25\%) were seropositive for NMOIgG [4]. This frequency accords with our clinical observations that less than $25 \%$ of patients with recurrent ON develop NMO. 
The absence of symptoms, signs and radiological evidence of brain involvement has traditionally served as a clinical criterion for making the diagnosis of NMO. However, we and others have recognized in recent years that some patients with longstanding NMO develop lesions (and more rarely symptoms) referable to the brainstem or brain [16]. Amongst these patients we have encountered some with otherwise typical NMO who developed brain MRI lesions judged as typical of MS. In addition, approximately $10 \%$ of individuals with NMO develop MRI lesions atypical for MS in the brainstem, hypothalamus or diffuse lesions in the cerebral hemispheres. Children are particularly overrepresented among those with atypical lesions. NMOIgG detection is proving to be of particular value for assigning the correct diagnosis.

The rheumatologic literature describes patients with neurological complications of lupus [28] or Sjögren's syndrome [29]. In both situations, longitudinally extensive myelitis, optic neuritis or both are noted. Disregarding the neurological manifestations, the patients of those reports generally lack sufficient clinical manifestations to make a formal diagnosis of lupus. Whether the myelitis in these conditions is distinct from NMO is unclear, but this question is now able to be addressed, by serological testing for NMO-IgG.

The clinical criteria used in Japan for the diagnosis of NMO are generally similar to the 1999 criteria developed at Mayo Clinic. However, in comparison with Mayo Clinic patients, the Japanese patients have an overall milder, though variable course. The descriptions of brain involvement on MRI in Japanese cases further suggested that Japanese "optic-spinal MS" differed from western NMO. The frequency of brain involvement in Japanese cases has not been reported to date. However, the results of our blinded analysis of coded serum samples from Japan not only confirmed the specificity of the marker for NMO, but strongly supports Japanese optic-spinal MS and NMO being the same entity [4]. The $58 \%$ seropositivity rate in Japanese patients with optic-spinal MS did not differ significantly from the $73 \%$ seropositivity rate in our larger group of North American patients with NMO.

\section{Potential pathogenicity of NMO-IgG}

Anecdotal reports and our own published observations document excellent responses to early plasma exchange therapy in patients with NMO. Of the 10 patients we treated by early plasmapheresis because of severe, steroid-refractory attacks of myelitis occurring in the context of NMO, 6 had moderate or marked improvement in neurological function within two weeks [30]. These observations are consistent with IgG being an effector of the basic pathology of the disorder.

The target antigen was identified at the molecular level first through demonstration by confocal microscopy with double immunofluorescence labeling that NMO patients' IgG bound to the abluminal face of penetrating microvessels, external to endothelium of spinal-cord microvessels (revealed by rabbit-IgGspecific for factor VIII), and to pia and subpial "mesh" in central nervous system tissues. Its colocalization with laminin around vessels [4] (Fig. 2) suggested that the NMO antigen was located at the blood-brain barrier, perhaps in foot processes of astrocytes. Surprisingly, the NMO antigen also was detected outside the nervous system, in the distal collecting tubules of the kidney and basolateral membranes of gastric parietal cells. Recognition that all these sites of NMO-IgG binding coincided with the distribution of the non-mercurialsensitive water channel aquaporin-4 (AQP4), led to formal demonstration that AQP4 is indeed the autoantigen recognized by NMO-IgG [31]. The perivascular distribution of immunoglobulins and a product of lytic complement activation (C9neo antigen) in spinal cord lesions of patients with NMO is remarkably similar to the pattern of immunofluorescence yielded by patients' IgG binding to mouse spinal cord tissue and supports a pathogenic role for NMO-IgG [19]. However, the lack of clinical involvement of stomach and kidney, and infrequency of brain involvement in NMO (all sites of high AQP4 expression) is not explained by the hypothesis that NMO is an aquaporinopathy.

Because approximately fifty percent of patients with NMO have autoantibodies that are commonly encountered in patients with other autoimmune disorders (e.g., antinuclear antibodies, thyroid antibodies) $[1,15]$, some may argue that NMO-IgG is similarly a non-specific indicator of autoimmunity in patients with NMO. However, it is our continuing experience that NMO-IgG is restricted to patients with NMO or a "high risk syndrome". The $27 \%$ rate of seronegativity amongst North American patients with clinically definite NMO might be explained by a species difference in the NMO antigens of rodent (used as our assay substrate) and man. However, organ-specific human autoantibodies detectable by indirect immunofluorescence are not in our experience restricted to primate tissues in reactivity [32]. Nevertheless, species polymorphisms are a 
major determinant of the antigenicity of plasma membrane cation channel antigens that interact with human autoantibodies in immunoprecipitation assays [33,34]. There are practical reasons for our preferred use of subprimate tissues as substrate for clinical immunostaining assays: 1) the high content of endogenous $\operatorname{IgG}$ in primate tissues cross-reacts with the anti-human IgG reagents used to detect NMO-IgG; and 2) the general lack of availability of fresh primate tissues, both monkey and human.

\section{Summary}

We have proved the clinical utility of NMO-IgG as a disease-specific biomarker, the first discovered for any demyelinating disease affecting the human CNS. Its antigen, AQP4, is the most abundant water channel in the central nervous system. NMO may represent the first example of a novel class of autoimmune channelopathy. Our observations to date suggest that the spectrum of NMO-related diseases is more extensive than previously appreciated. In our present clinical practice we evaluate patients serologically on a routine basis to aid early distinction of NMO from MS, to enable prompt initiation of most appropriate IgGdepleting and immunosuppressant treatment for NMO, rather than the immunomodulatory therapies that are promoted for MS.

\section{References}

[1] D.M. Wingerchuk, W.F. Hogancamp, P.C. O'Brien and B.G. Weinshenker, The clinical course of neuromyelitis optica (Devic's syndrome), Neurology 53 (1999), 1107-1114.

[2] B.A.C. Cree, D.S. Goodin and S.L. Hauser, Neuromyelitis optica [Review], Seminars in Neurology 22 (2002), 105-122.

[3] D.M. Wingerchuk, Neuromyelitis Optica: Current Concepts, Frontiers in Bioscience 9 (2004), 834-840.

[4] V.A. Lennon, D.M. Wingerchuk, T.J. Kryzer et al., A serum autoantibody marker of neuromyelitis optica: Distinction from multiple sclerosis, Lancet 364 (2004), 2106-2112.

[5] F. Gault, De la neuromyelite optique aigue, Thesis, Lyon: 1894.

[6] C. Devic, Myelite subaigue compliquee de nevrite optique, Bull Med 35 (1894), 18-30.

[7] G.M. Beck, A case of diffuse myelitis associated with optic neuritis, Brain 50 (1927), 687-703.

[8] J. Kira, Multiple sclerosis in the Japanese population, Lancet Neurology 2 (2003), 117-127.

[9] T. Misu, K. Fujihara, I. Nakashima et al., Pure optic-spinal form of multiple sclerosis in Japan, Brain 125 (2002), 24602468 .
[10] R.N. Mandler, W. Ahmed and J.E. Dencoff, Devic's neuromyelitis optica: a prospective study of seven patients treated with prednisone and azathioprine, Neurology 51 (1998), 12191220 .

[11] D. McAlpine, Familial neuromyelitis optica: Its occurrence in identical twins, Brain 61 (1938), 430-448.

[12] L.T. Ch'ien, M.O. Medeiros, J.J. Belluomini et al., Neuromyelitis optica (Devic's syndrome) in two sisters, Clinical Electroencephalography 13 (1982), 36-39.

[13] M. Keegan and B. Weinshenker, Familial Devic's disease, Canadian Journal of Neurological Sciences. 27 (2000), S57S58.

[14] K. Yamasaki, I. Horiuchi, M. Minohara et al., HLADPB $1 * 0501$-associated opticospinal multiple sclerosis: clinical, neuroimaging and immunogenetic studies, Brain 122(Pt 9) (1999), 1689-1696.

[15] J.I. O'Riordan, H.L. Gallagher, A.J. Thompson et al., Clinical, CSF, and MRI findings in Devic's neuromyelitis optica, Journal of Neurology, Neurosurgery \& Psychiatry. 60 (1996), 382-387.

[16] S.J. Pittock, V.A. Lennon, K. Krecke et al., Brain abnormalities in patients with neuromyelitis optica, Arch Neurol 63 (2006), 390-396.

[17] D.M. Wingerchuk and B.G. Weinshenker, Neuromyelitis optica - Clinical predictors of a relapsing course and survival, Neurology 60 (2003), 848-853.

[18] R.N. Mandler, L.E. Davis, D.R. Jeffery and M. Kornfeld, Devic's neuromyelitis optica: a clinicopathological study of 8 patients, Ann Neurol 34 (1993), 162-168.

[19] C.F. Lucchinetti, R.N. Mandler, D. McGavern et al., A role for humoral mechanisms in the pathogenesis of Devic's neuromyelitis optica, Brain. 125 (2002), 1450-1461.

[20] C.F. Lucchinetti, W. Bruck, J. Parisi et al., Heterogeneity of multiple sclerosis lesions: Implications for the pathogenesis of demyelination, Ann Neurol 47 (2000), 707-717.

[21] M.K. Storch, A. Stefferl, U. Brehm et al., Autoimmunity to myelin oligodendrocyte glycoprotein in rats mimics the spectrum of multiple sclerosis pathology, Brain Pathol 8 (1998), 681-694.

[22] R. Gold and C. Linington, Devic's disease: bridging the gap between laboratory and clinic, Brain 125 (2002), 1425-1427.

[23] J.A. Simpson, Myasthenia gravis: A new hypothesis, Scott Med J 5 (1960), 419-436.

[24] V.A. Lennon, E.H. Lambert, S. Whittingham and V. Fairbanks, Autoimmunity in the Lambert-Eaton myasthenic syndrome, Muscle \& Nerve 5 (1982), S21-S25.

[25] V.A. Lennon, Paraneoplastic autoantibodies: the case for a descriptive generic nomenclature, Neurology 44 (1994), 22362240 .

[26] B. Weinshenker, D. Wingerchuk, S. Vukusic et al., NMO-IgG predicts relapse following longitudinally extensive transverse myelitis, Ann Neurol 59 (2006), 566-569.

[27] I. Pirko, L.K. Blauwet, T.G. Lesnick and B.G. Weinshenker, The natural history of recurrent optic neuritis, Arch Neurol 61 (2004), 1401-1405.

[28] F. Bonnet, P. Mercie, P. Morlat et al., Devic's neuromyelitis optica during pregnancy in a patient with systemic lupus erythematosus, Lupus 8 (1999), 244-247.

[29] A. Mochizuki, A. Hayashi, S. Hisahara and S. Shoji, Steroidresponsive Devic's variant in Sjogren's syndrome, Neurology 54 (2000), 1391-1392.

[30] B.M. Keegan, A.A. Pineda, R.L. McClelland et al., Plasma exchange for severe attacks of CNS demyelination: predictors of response, Neurology 58 (2002), 143-146. 
[31] V.A. Lennon, T.J. Kryzer, S.J. Pittock et al., IgG marker of optic-spinal MS binds to the aquaporin 4 water channel, $J$ Exp Med 202 (2005), 473-477.

[32] J.L. Smith, J.C. Finley and V.A. Lennon, Autoantibodies in paraneoplastic cerebellar degeneration bind to cytoplasmic antigens of Purkinje cells in humans, rats and mice and are of multiple immunoglobulin classes, J. Neuroimmunol. 18 (1988), 37-48.
[33] E.H. Lambert and V.A. Lennon, Selected IgG rapidly induces Lambert-Eaton myasthenic syndrome in mice: Complement independence and EMG abnormalities, Muscle \& Nerve. 11 (1988), 1133-1145.

[34] V.A. Lennon and G.E. Griesmann, Evidence against acetylcholine receptor having a main immunogenic region as target for autoantibodies in myasthenia gravis, Neurology 39 (1989), 1069-1076. 


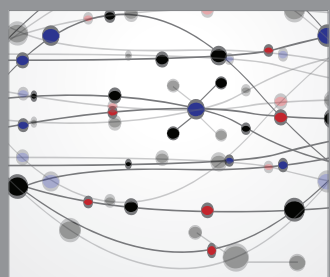

The Scientific World Journal
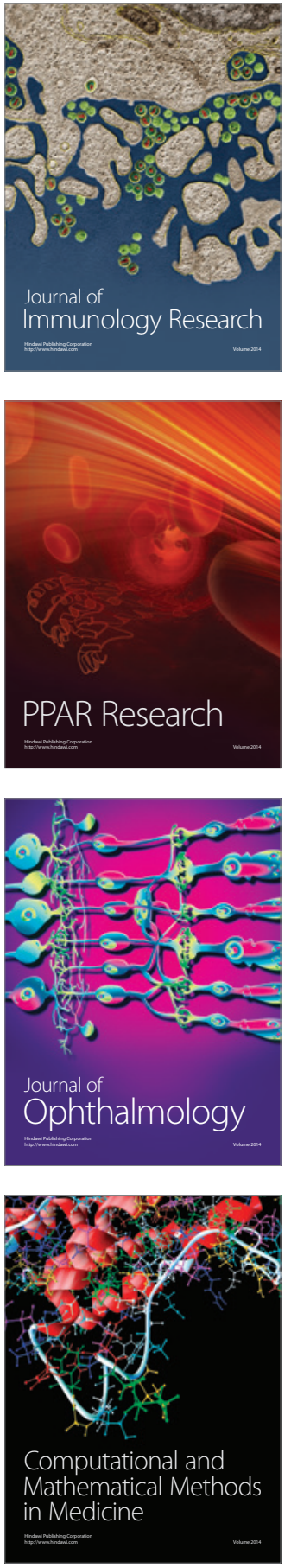

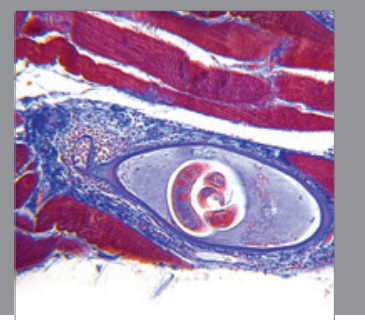

Gastroenterology

Research and Practice
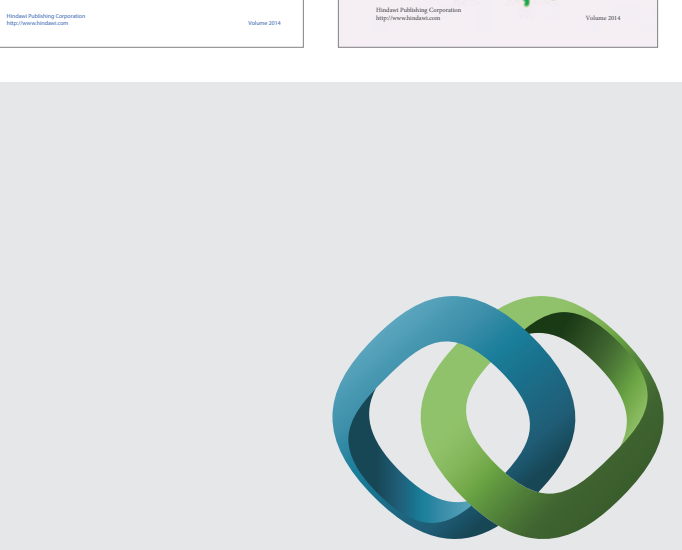

\section{Hindawi}

Submit your manuscripts at

http://www.hindawi.com
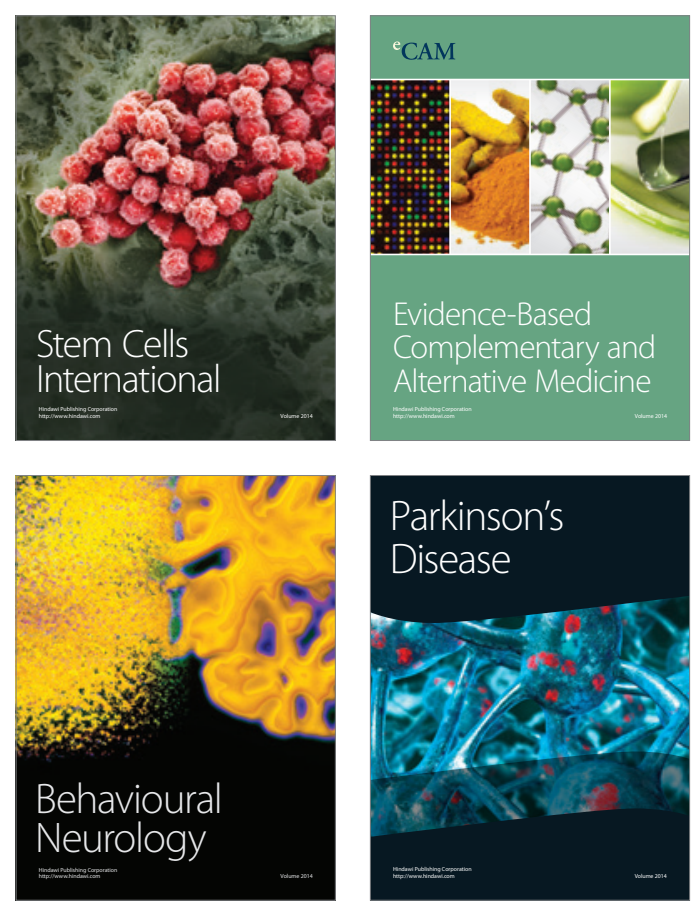

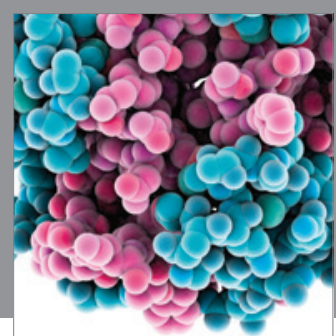

Journal of
Diabetes Research

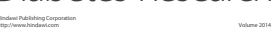

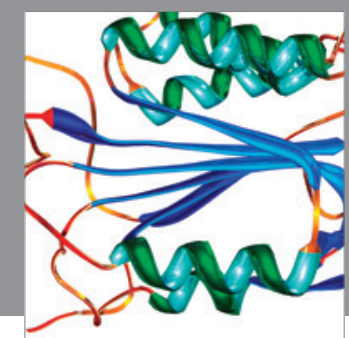

Disease Markers
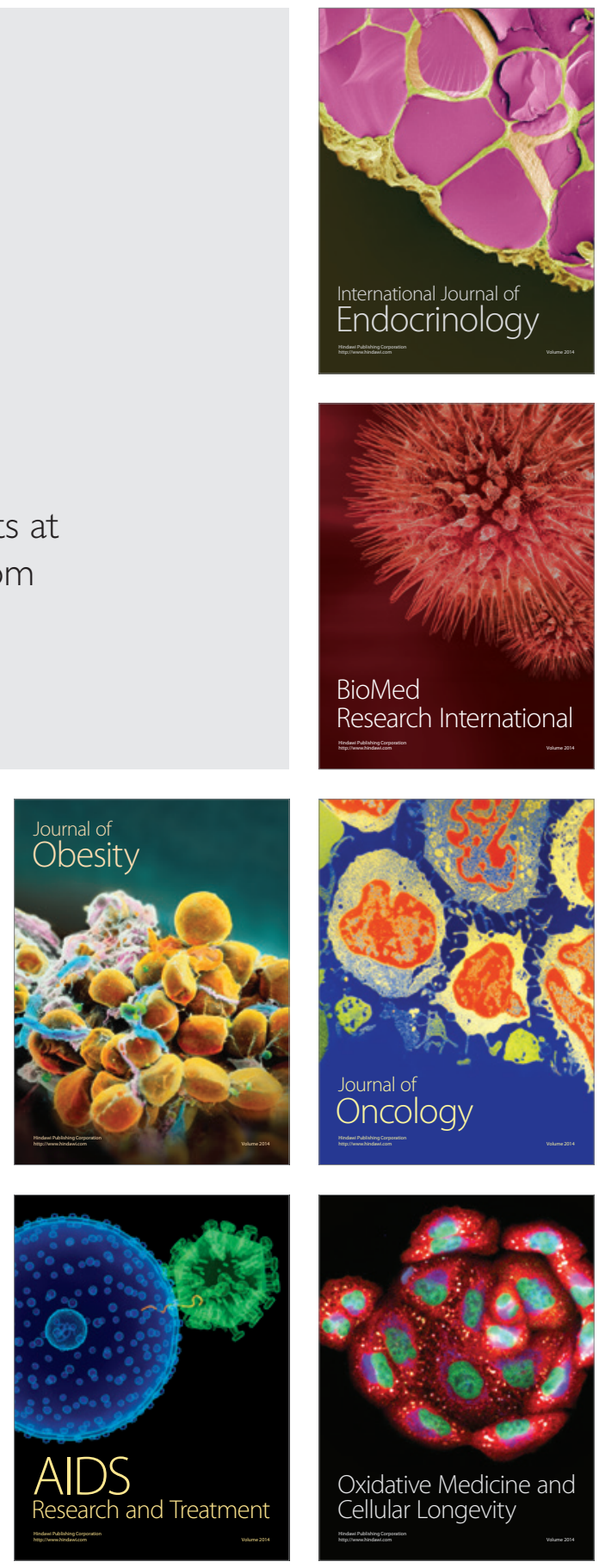